\title{
Video Article \\ Isolation and Culture of Pulmonary Endothelial Cells from Neonatal Mice
}

\author{
Magdalena Sobczak ${ }^{1}$, Jillian Dargatz ${ }^{1}$, Magdalena Chrzanowska-Wodnicka ${ }^{1}$ \\ ${ }^{1}$ Blood Research Institute, BloodCenter of Wisconsin
}

Correspondence to: Magdalena Chrzanowska-Wodnicka at Magdalena.Wodnicka@bcw.edu

URL: https://www.jove.com/video/2316

DOI: doi:10.3791/2316

Keywords: Cellular Biology, Issue 46, Endothelium, lung, microvascular cells, mouse, isolation, angiogenesis, vascular permeability, adherens junctions

Date Published: 12/14/2010

Citation: Sobczak, M., Dargatz, J., Chrzanowska-Wodnicka, M. Isolation and Culture of Pulmonary Endothelial Cells from Neonatal Mice. J. Vis. Exp. (46), e2316, doi:10.3791/2316 (2010).

\section{Abstract}

Endothelial cells provide a useful research model in many areas of vascular biology. Since its first isolation ${ }^{1}$, human umbilical vein endothelial cells (HUVECs) have shown to be convenient, easy to obtain and culture, and thus are the most widely studied endothelial cells. However, for research focused on processes like angiogenesis, permeability or many others, microvascular endothelial cells (ECs) are a much more physiologically relevant model to study ${ }^{2}$. Furthermore, ECs isolated from knockout mice provide a useful tool for analysis of protein function ex vivo. Several approaches to isolate and culture microvascular ECs of different origin have been reported to date ${ }^{3-7}$, but consistent isolation and culture of pure ECs is still a major technical problem in many laboratories. Here, we provide a step-by-step protocol on a reliable and relatively simple method of isolating and culturing mouse lung endothelial cells (MLECs). In this approach, lung tissue obtained from 6- to 8-day old pups is first cut into pieces, digested with collagenase/dispase (C/D) solution and dispersed mechanically into single-cell suspension. MLECS are purified from cell suspension using positive selection with anti-PECAM-1 antibody conjugated to Dynabeads using a Magnetic Particle Concentrator (MPC). Such purified cells are cultured on gelatin-coated tissue culture (TC) dishes until they become confluent. At that point, cells are further purified using Dynabeads coupled to anti-ICAM-2 antibody. MLECs obtained with this protocol exhibit a cobblestone phenotype, as visualized by phase-contrast light microscopy, and their endothelial phenotype has been confirmed using FACS analysis with anti-VE-cadherin ${ }^{8}$ and anti-VEGFR2 ${ }^{9}$ antibodies and immunofluorescent staining of VE-cadherin. In our hands, this two-step isolation procedure consistently and reliably yields a pure population of MLECs, which can be further cultured. This method will enable researchers to take advantage of the growing number of knockout and transgenic mice to directly correlate in vivo studies with results of in vitro experiments performed on isolated MLECs and thus help to reveal molecular mechanisms of vascular phenotypes observed in vivo.

\section{Video Link}

The video component of this article can be found at https://www.jove.com/video/2316/

\section{Protocol}

\section{Preparing anti-PECAM-1 antibody-conjugated magnetic beads (Dynabeads)}

1. Prepare $0.1 \%$ BSA in PBS by mixing $50 \mathrm{mg}$ BSA in $50 \mathrm{ml}$ PBS using a vortex until BSA dissolves. Sterilize by filtering through $0.22 \mu \mathrm{m}$ syringe filter. Store at $4^{\circ} \mathrm{C}$.

2. In the TC hood, transfer $200 \mu \mathrm{l}$ of well resuspended sheep anti-rat IgG Dynabeads into a $1.5 \mathrm{ml}$ Eppendorf tube and wash the beads: Mount tube on MPC and let sit for about $1 \mathrm{~min}$ to allow the beads to sediment. Aspirate supernatant, remove the tube from MPC and resuspend the beads in $1 \mathrm{ml}$ of sterile $0.1 \%$ BSA/PBS. Pipette up and down to resuspend beads.

3. Repeat wash three more times, for a total of four washes.

4. Remove tube from MPC and resuspend the beads in $500 \mu \mathrm{l}$ of $0.1 \% \mathrm{BSA} / \mathrm{PBS}$.

5. Add $10 \mu \mathrm{l}$ rat anti-mouse PECAM-1 (CD-31) antibody to the tube.

6. Tumble overnight at $4^{\circ} \mathrm{C}$ in the cold room or $2 \mathrm{hrs}$ at room temperature.

7. Wash the beads with sterile $0.1 \%$ BSA/PBS as described in step 1.2 four times.

8. Remove tube from MPC and resuspend the beads in $200 \mu \mathrm{l} 0.1 \%$ BSA/PBS. Store anti-PECAM- 1 antibody-conjugated Dynabeads at $4{ }^{\circ} \mathrm{C}$ for up to 1 month.

\section{Isolating mouse pulmonary endothelial cells from neonatal mice}

Prior to proceeding with the tissue purification protocol, IACUC Committee approval of the procedure is required.

1. Prepare the following:

- $15 \mathrm{ml}$ of $1 \mathrm{mg} / \mathrm{ml} \mathrm{C/D}$ solution in DMEM. Filter with $0.22 \mu \mathrm{m}$ syringe filter and warm to $37^{\circ} \mathrm{C}$.

- $50 \mathrm{ml}$ conical tube with $15 \mathrm{ml}$ DMEM, store on ice 
- Isolation Media (IM) containing $20 \%$ FBS and $1 \times$ penicillin/streptomycin in DMEM

- $2 \%$ gelatin. Mix $2 \mathrm{~g}$ of bovine skin gelatin with $100 \mathrm{ml} \mathrm{dd}$ water, autoclave 15 minutes and store at $4^{\circ} \mathrm{C}$. Warm to $37^{\circ} \mathrm{C}$ to liquefy before coating TC flasks.

2. Anesthetize three 6-8 day old pups using an IP injection of Ketamine $(100 \mathrm{mg} / \mathrm{kg})$ and xylazine $(10 \mathrm{mg} / \mathrm{kg})$. Check for effectiveness of anesthesia and dissect animals one by one, as follows: Pin pups to board, soak skin with $70 \%$ Ethanol and remove skin from the chest area with sterile dissection scissors. Excise the rib cage to expose lungs.

3. Aseptically excise individual lung lobes, being careful not to dissect the bronchi and any visible connective tissue around the lungs. Combine all lungs in ice-cold DMEM. Euthanize the pups.

4. Working in the TC hood, remove lung lobes from DMEM, place in a $100 \mathrm{~mm}$ TC dish and aspirate excess DMEM.

5. Using sterile scissors, mince the tissue finely by cutting $\sim 100$ times. Transfer to $15 \mathrm{ml}$ warm C/D solution for enzymatic digestion. Incubate 45 minutes at $37^{\circ} \mathrm{C}$ on a rotator.

6. In the TC hood, aspirate the digested tissue suspension into to a $20 \mathrm{ml}$ syringe with $14 \mathrm{~g}$ cannula attached and triturate clumps into a single cell suspension, at least 12 times.

7. Pass the tissue suspension through a $70 \mu \mathrm{m}$ cell strainer and wash the strainer with $15 \mathrm{ml} \mathrm{IM}$ to stop digestion.

8. Pellet cell suspension by centrifugation at $400 \mathrm{x} g$ for 5 minutes.

9. Aspirate supernatant and resuspend pellet in $3 \mathrm{ml} 0.1 \% \mathrm{BSA} / \mathrm{PBS}$.

10. Transfer cell suspension to $5 \mathrm{ml}$ round-bottom polystyrene tube and add $22.5 \mu \mathrm{l}$ anti-PECAM-1 antibody-conjugated Dynabeads. Tumble at room temperature for 12 minutes.

11. Coat a T75 flask with $2 \mathrm{~mL} 2 \%$ gelatin and aspirate excess gelatin. Allow gelatin to dry inside the TC hood for about 30 minutes prior to plating cells.

12. After bead incubation is complete (step 2.10), split cell suspension equally into three Eppendorf tubes and mount on the MPC.

13. When the beads have sedimented using the MPC $(\sim 1 \mathrm{~min})$, collect supernatant, remove tube from MPC, wash beads with $\sim 1 \mathrm{ml} 0.1 \%$ BSA/ PBS, and then remount on the MPC.

14. Repeat wash four times (total 5 washes)

15. Resuspend beads in $1 \mathrm{ml}$ of VascuLife with EnGS-Mv Life Factors Kit and 1x penicillin/streptomycin (VL) per tube, mixing well.

16. Plate on a pre-coated T75 flask and bring total volume in each flask to $10 \mathrm{ml}$ with VL.

17. Change media the following day, allow 1 full day of growth, and then change half of the media every other day. When cells are $>70-80 \%$ confluent or more (usually after 3-4 days of culture), they can be sorted with anti-ICAM-2 Dynabeads.

\section{Preparing anti-ICAM-2 antibody-conjugated Dynabeads}

1. Anti-ICAM-2 antibody-conjugated Dynabeads are used to further purify cells that have been selected with anti-PECAM-1 antibodyconjugated Dynabeads and cultured until confluent. This procedure is performed as described for anti-PECAM-1 antibody-conjugated Dynabeads (1, above), except that anti-mouse ICAM-2 (CD-102) antibody is used instead of anti-PECAM-1 antibody.

2. Anti-ICAM-2 antibody-conjugated Dynabeads can be stored at $4^{\circ} \mathrm{C}$ for up to 1 month.

\section{Sorting Mouse Lung Endothelial Cells with anti-ICAM-2 Dynabeads}

1. Coat T75 tissue culture flask with $2 \%$ gelatin.

2. Aspirate the media from the confluent T75 flask with cells and wash with $8 \mathrm{ml}$ PBS.

3. Aspirate PBS, add $2 \mathrm{ml} 0.05 \%$ Trypsin/EDTA and let cells detach (about 5 minutes). Tap flasks lightly to aid detaching.

4. When cells have detached, add $2 \mathrm{ml} \mathrm{IM}$ to inhibit trypsin and scrape cells to detach any remaining adherent cells. Transfer cell suspension to a $15 \mathrm{ml}$ tube and spin down for $5 \mathrm{~min}$ at $400 \mathrm{x} \mathrm{g}$.

5. Aspirate the media and resuspend cell pellet in $2 \mathrm{ml} 0.1 \% \mathrm{BSA} / \mathrm{PBS}$.

6. Transfer to a $5 \mathrm{ml}$ polystyrene round-bottom tube and add $10 \mu \mathrm{l}$ anti-ICAM-2 Dynabeads. Tumble for 12 minutes at RT.

7. Split cell suspension into two $1.5 \mathrm{ml}$ Eppendorf tubes and mount on MPC.

8. Aspirate supernatant after beads have adhered for one minute. Remove tubes from MPC and resuspend each tube in $1 \mathrm{ml} 0.1 \%$ BSA/PBS. Remount on MPC.

9. Repeat wash four times (total of 5 washes).

10. Resuspend each tube with $1 \mathrm{ml} \mathrm{VL}$ and perform a cell count.

11. Plate cells at $250-350 \mathrm{~K}$ cells per T25 flask and/or $750 \mathrm{~K}-1$ million cells per T75 flask.

12. Bring volume to $5 \mathrm{ml} \mathrm{VL}$ in T25 flasks, and $10 \mathrm{ml}$ in T75 flasks.

13. Change media every other day. Cells can be used for experiments when culture reaches about $80 \%$ confluency, usually in $2-3$ days.

\section{Representative Results}

Typically, after 6-7 days from the initial preparation of the cells, we are able to obtain about $1.2-1.5 \times 10^{6}$ pulmonary endothelial cells from three 6-8 day old pups. Cells display typical "cobblestone" morphology under light microscopy and show VE-cadherin (CD144) staining at cell-cell junctions, which is characteristic for endothelial cells (Figure 1). Majority of MLECs express VE-cadherin and VEGFR2 as demonstrated by FACS analysis (Figure 2). Usually, we use them for experiments within two weeks after the initial MLEC isolation. 


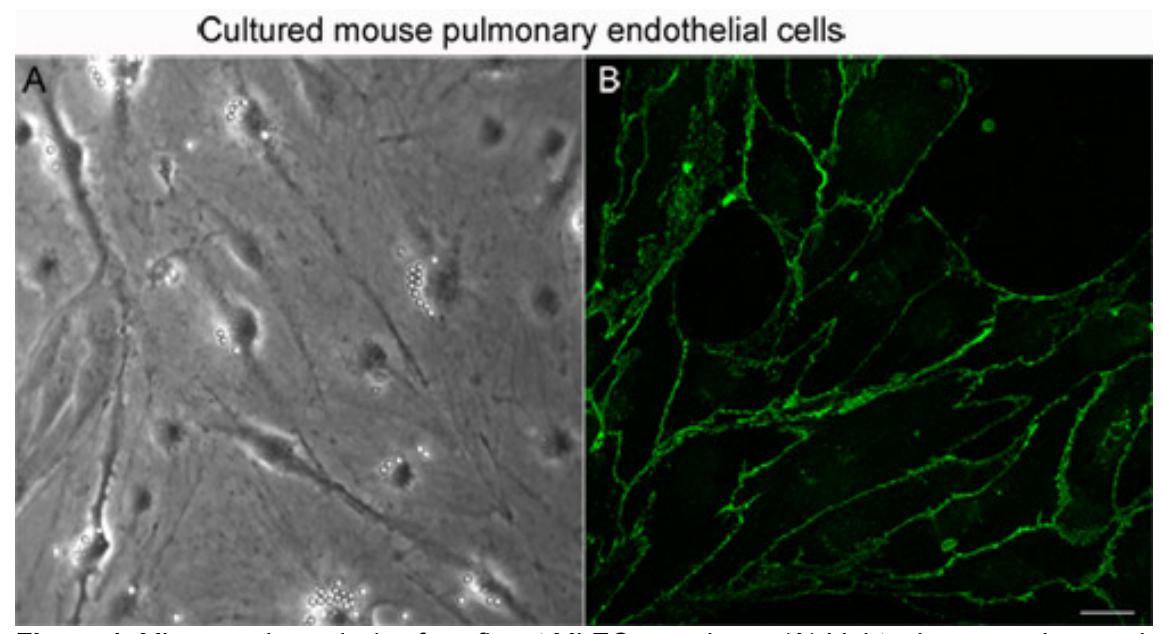

Figure 1. Microscopic analysis of confluent MLEC monolayer. (A) Light microscopy image shows cobblestone morphology of cultured cells typical for endothelial cells. Uniform round structures located in the perinuclear area of many cells are the magnetic beads used for MLEC isolation. (B) Endothelial-specific VE-cadherin is localized at the cell-cell junctions as shown using confocal microscopy. Bar is representative of $20 \mu \mathrm{m}$.

\section{FACS analysis of VE-cadherin and VEGFR2 surface expression}
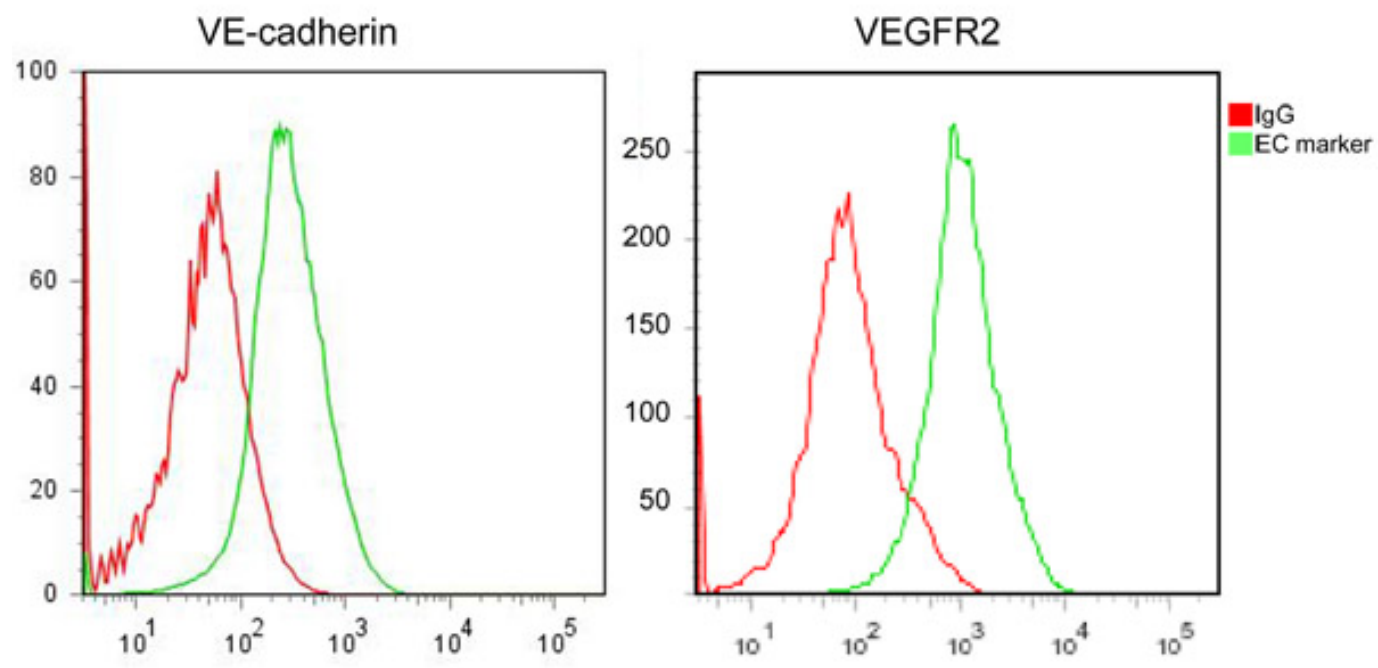

Figure 2. FACS analysis of cultured MLECs labeled with antibodies specific for endothelial-specific markers: VE-cadherin (CD144) or VEGFR2, as indicted, followed by phycoerythrin-conjugated secondary antibody, confirms endothelial identity of isolated MLECs. Red line indicates isotype-specific control, green line indicates anti -VE-cadherin or anti-VEGFR2 - specific-lgG.

\section{Discussion}

Microvascular ECs have proven to be a useful model in many areas of vascular biology and are believed to be more physiologically relevant to study (e.g. angiogenesis) than widely studied HUVECs ${ }^{3}$. Previously, it has been reported that microvascular ECs can be obtained from kidney, heart, skin, retina, brain, gliomas, adipose tissue and also lung ${ }^{2,4-7,10,11}$. However, a consistent and reliable method for isolation of microvascular ECs is still required. The procedure presented here is a modification of a previously published protocol ${ }^{2}$; it differs from most published procedures in that it uses pups instead of adult animals. This is crucial for the isolation success as cells from young animals have a higher proliferation potential and cultures derived from their tissues tend to yield higher number of cells. One week old animals seem to be optimal, but younger mice (4 day-old) can also be used. Also, higher or lower numbers of pups can be used if required, as we have been successful in isolating MLECs from one pup. However, while scaling up or down the isolation process, cell plating density must remain unchanged, as this is also critical for cell proliferation. The 2-step purification process of MLECs using magnetic beads conjugated first to antiPECAM-1 and than anti-ICAM-2 antibodies is much more efficient at obtaining pure EC cultures than the previously described single-step purification procedures. This protocol eliminates the need to use very laborious manual techniques, gradient centrifugation and less efficient FACS sorting for discarding contaminating cells such as fibroblasts, blood cells and smooth muscle cells. The resulting MLEC population can be used for in vitro analysis of angiogenic responses, vascular permeability and leukocyte transmigration, wound healing as well as biochemical analysis of signaling pathways. If required, MLECs can be plated on different surfaces such as fibronectin or gelatin-coated coverslips for immunofluorescence or electrode arrays for trans-endothelial resistance (TER) measurements. Obtained results can be directly compared to the phenotypes observed in vivo, which is especially important in the context of the growing number of knockout and transgenic mouse lines. 
Successful implementation of this method for in vitro analysis of cellular mechanisms underlying defects observed in vivo, has already been presented $^{12}$.

\section{Disclosures}

Experiments on animals were performed in accordance with the guidelines and regulations set forth by Medical College of Wisconsin IACUC Committee under protocol AUA\#00001206.

\section{Acknowledgements}

This research was supported in part by American Heart Association grant 0950118G.to M.C-W.

\section{References}

1. Maruyama, Y. The human endothelial cell in tissue culture. Z Zellforsch Mikrosk. Anat. 60, 69-79 (1963).

2. Lim, Y.C., Luscinskas, F.W. Isolation and culture of murine heart and lung endothelial cells for in vitro model systems. Methods Mol Biol. 341:141-154 (2006).

3. Aird, W.C. Phenotypic heterogeneity of the endothelium: I. Structure, function, and mechanisms. Circ Res. 100, 158-73 (2007).

4. Fehrenbach, M.L., Cao, G., Williams, J.T., Finklestein, J.M., Delisser, H.M. Isolation of murine lung endothelial cells. Am J Physiol Lung Cell Mol Physiol. 296:L1096-103 (2009).

5. Miebach, S., Grau, S., Hummel, V., Rieckmann, P., Tonn, J.C., Goldbrunner, R.H. Isolation and culture of microvascular endothelial cells from gliomas of different WHO grades. J. Neurooncol. 76, 39-48 (2006).

6. Demeule, M., Labelle, M., Régina, A., Berthelet, F., Béliveau, R. Isolation of endothelial cells from brain, lung, and kidney: expression of the multidrug resistance P-glycoprotein isoforms. Biochem. Biophys. Res. Commun. 281, 827-34 (2001).

7. Kajimoto, K., Hossen, N., Hida, K., Ohga, N., Akita, H., Hyodo, M., Hida, Y., Harashima, H. Isolation and culture of microvascular endothelia cells from murine inguinal and epididymal adipose tissues. J. Immunol. Methods. 357, 43-50 (2010).

8. Breviario, F., Caveda, L., Corada, M., Martin-Padura, I., Navarro, P., Golay, J., Introna, M., Gulino, D., Lampugnani, M.G., Dejana, E. Functional properties of human vascular endothelial cadherin (7B4/cadherin-5), an endothelium-specific cadherin. Arterioscler Thromb Vasc Biol. 15, 1229-39 (1995).

9. Zachary, I., Gliki, G. Signaling transduction mechanisms mediating biological actions of the vascular endothelial growth factor family. Cardiovasc Res. 49, 568-581 (2001).

10. Diglio, C.A., Grammas, P., Giacomelli, F., Wiener, J. Rat heart-derived endothelial and smooth muscle cell cultures: isolation, cloning and characterization. Tissue Cell. 20, 477-92 (1988).

11. Petzelbauer, P., Bender, J.R., Wilson, J., Pober, J.S. Heterogeneity of dermal microvascular endothelial cell antigen expression and cytokine responsiveness in situ and in cell culture. J Immunol. 151, 5062-72 (1993).

12. Chrzanowska-Wodnicka, M., Kraus, A.E., Gale, D., White GC $2^{\text {nd }}$, Vansluys J. Defective angiogenesis, endothelial migration, proliferation, and MAPK signaling in Rap1b-deficient mice. Blood. 111, 2647-56 (2008). 